\title{
Article \\ The Role of General Practitioners in Suicide Prevention-What You Said and Did Then Actually Saved My Life
}

\author{
Marte Styrvold ${ }^{1}$ and Tine K. Grimholt ${ }^{1,2, *}$ \\ 1 Department of Acute Medicine, Oslo University Hospital, 0424 Oslo, Norway; mstyrvold@gmail.com \\ 2 Faculty of Health, VID Specialized University, 0319 Oslo, Norway \\ * Correspondence: Tinegrim@yahoo.no; Tel.: +47-9012-0011
}

Citation: Styrvold, M.; Grimholt, T.K The Role of General Practitioners in Suicide Prevention-What You Said and Did Then Actually Saved My Life. Reports 2021, 4, 23. https://doi.org/ $10.3390 /$ reports 4030023

Academic Editor: Toshio Hattori

Received: 21 May 2021

Accepted: 3 August 2021

Published: 6 August 2021

Publisher's Note: MDPI stays neutral with regard to jurisdictional claims in published maps and institutional affiliations.

Copyright: (c) 2021 by the authors. Licensee MDPI, Basel, Switzerland. This article is an open access article distributed under the terms and conditions of the Creative Commons Attribution (CC BY) license (https:// creativecommons.org/licenses/by/ $4.0 /)$.

\begin{abstract}
Background: General practitioners (GPs) have an important role in the prevention of suicidal behavior. The purpose of this study was to explore their views and experiences in identifying and assessing suicidal ideation. Methods: Ten GPs were recruited through convenience sampling based on accessibility, interest and willingness to participate. In-depth interviews were carried out, and the results were transcribed verbatim. Aspects of experiences with suicidal patients emerged through the thematic analysis process. Results: The GPs described the varied clinical picture when patients presented in their office. How they identified depressive symptoms apart from originally somatic complaints included forming a trusting relationship and addressing suicide ideation. They described customized interventions as well as obstacles and factors that facilitated communication, including time, their own personal traits, patient's disclosure and organizational barriers. Conclusions: The levels of the suicidal process among patients in general practice vary greatly. GPs adjust their appraisals to profoundly understand and intervene to prevent a crisis of escalation into subsequent suicidal behavior.
\end{abstract}

Keywords: general practitioners; suicide prevention; qualitative method

\section{Introduction}

The general practitioner (GP) role and potential have been pointed out as important in suicide prevention [1]. To exploit this potential, it is particularly important to understand how they work with suicidal patients in general practice.

However, previous research has, to a large extent, focused on the amount of fatal cases that consulted their GP before they attempted or died by suicide [2] and thus focused on a low frequency of consultations which, in turn, excluded knowledge of the total prevention potential in general practice and overlooked the primary prevention focus.

Already in the early 1980s, Sakinfosky underlined how the family doctors had a role to play at all levels of suicide prevention to act effectively in their own offices, where they routinely considered depression and suicide potential in their patients [3].

Annually, a high number of patients consult their GPs. In Norway in 2019, there were more than 14 million consultations in general practice in age groups above 16 years [4], and 1.5 million were due to mental illness or mental health problems [4]. However, most of these patients never engaged in suicidal behavior. The suicide rates in Norway have been approximately 550 per year. Of these, not all consulted their GPs at any time preceding the suicide. A review demonstrated that an average of $55 \%$ of people that committed suicide never went to see their GPs in the month before the suicide [5]. In addition, the fact that many of the last consultations with the GPs before a suicide attempt were due to somatic conditions [6] underline that it is impossible to predict which patient will engage in suicidal behavior. Furthermore, there is reason believe that interventions carried out in general practice are preventive when the suicidal process is interrupted at an early stage, thus hindering suicidal actions. 
The aim of this study was to gain empirical knowledge by exploring the GPs' clinical experiences and providing a broader understanding for educational initiatives as well as policy makers.

\section{Materials and Methods}

\subsection{Setting and Recruitment}

The GPs in the Southeast Region of Norway were chosen strategically to include both short and long experience from the general medicine and gender perspectives. Five men and five women were interviewed, with the ages ranging from 29 to 64 years (experience corresponded with age), and half had worked in psychiatry or had education in cognitive behavioral therapy as part of their specialist training. Participants were recruited via colleagues, the snowballing method, and being approached by email or text messages. The participants did not know the interviewer. The study was approved by the Personal Protection Agency at Oslo University Hospital. All participants were informed and signed written consent. Strict confidentiality to avoid recognition of patients was kept in transcripts and in the manuscript.

\subsection{Interviews}

The research questions were formulated to provide the researcher with an understanding of the meaning of the GPs' experiences and to explore phenomena. The preliminary questions were therefore formulated broadly and openly to be dynamic, flexible and possible to change, as the thematic frame might change during data gathering and if unknown phenomena occurred.

The preliminary research questions were the following:

What is the role of the GP in suicide prevention?

What are the GPs doing to prevent suicidal behavior among their patients?

How do the GPs experience meeting with suicidal patients?

What do they experience as negative and difficult?

What will they advise other GPs?

A considerable part of the project needed to be worked through before interviewing. It was therefore necessary that the interviewer was familiar with concepts and theoretical understanding from suicidology and general practice. The interviewer had extensively reviewed the related literature. It was also important to follow up interesting ideas and see the new dimensions, which were not anticipated in advance of the interviews [7].

The participants were encouraged to talk about the issues pertinent to the research questions in one-on-one in-depth interviews. The GPs talked freely and gave narratives about their experiences, perceptions and feelings surrounding topics of suicidality in their practice.

After the first five interviews (two men and three women), all the records were transcribed verbatim, and the main themes were added to the last five interviews to elaborate on topics that were revealed during the first phase of the research process.

This sequential interim analysis allowed the researcher to go back and refine the questions, develop hypotheses and pursue emerging avenues of inquiry in greater depth during the second phase [8].

The second part of the process also allowed for further investigation to clarify, verify and familiarize the findings and, as outlined by Murphy, search for inconsistent data and try to falsify the phenomena introduced by the first respondents by revealing opposite data $[7,9]$. The data collection was terminated when the responses indicated that saturation was achieved.

\subsection{Data Analysis}

All interviews were transcribed verbatim. They were analyzed with five stages of data analyses using the framework approach: familiarization, identifying a thematic framework, indexing, charting and finally mapping and interpretation [8]. To obtain the meaning in the 
text after the records were transcribed, the materials included notes from the interviews that were read to obtain the first trend of the greater picture.

\section{Results}

The key themes that emerged from the data were presented and supported by illustrating quotes from the transcripts. The findings described different pathways or steps and measures performed by GPs in the process from the recognition of symptoms and identification of suicidality to specific interventions.

The themes are described in the following order: initial diagnosis, symptoms, cues, concerns and the patient's disclosure of problems. The recognition and clarifying phase involved tuning into the essence of the problem and forming an alliance, followed by identification of suicide ideation and intervention strategies.

The GPs' narratives formed a picture of the varied and diverging problems and struggles among their patients, from the ones that did not recognize that their symptoms indicated depression and, on the contrary, patients that were explicit about constantly thinking of ending their lives. The underlying and triggering causes ranged from serious traumatic childhood exposure to different sorts of transient life crises.

\subsection{Initial Diagnosis}

When the patient entered the consultation, the actual reason was not always specified. One example was when a patient scheduled an appointment with the secretary because of a somatic diagnosis instead of revealing their actual problems because they did not want to disclose their personal life or feared stigma. When they arrived at the doctor's office, the patient said that this diagnosis was not their actual problem. In these cases, the GPs scheduled a new appointment themselves so the patient did not have to talk with or disclose this to the secretary.

\subsection{Symptoms}

The reason for consulting the GP is not predefined with a diagnosis when a patient arrives with a variety of symptoms like fatigue, sleeplessness or headaches. Patients' beliefs about their symptoms do not always align with the GP and might influence their decisions to consult or how they present their problems. One common example that was mentioned was fatigue which, for instance, can be a symptom of depression or anemia or be related to negative life events.

\subsubsection{Unrecognized Symptoms}

Some patients did not understand their symptoms and were worried. One of the GPs gave an illustrative narrative about unrecognized symptoms:

I met a very interesting man for about $x$ years ago, I still have him on my list, that's why I remembered him. He received a severance package, was fired from work or pre retired or something. Came and complained about ..(thinking) ... very straight forward kind of type without any reflections about emotions or something like that. And he came and explained how he was doing, I listened and asked a bit.. and at the end I said to him: Do you feel sad and down? "Yes, I do" he said, "Are you depressed?" "O yes.. (curses).. yes so that is what it is.."

So, he understood that this was an emotional reaction he had and that he could live with, you know ... he was afraid that he had gotten cancer.

A similar example was given by another GP:

I recently had a patient that came to me, for not psychiatry, but I started to suspect that there was "some psychiatry here". A smart man, in fulltime job, in his 50s, but as I understood was a little ... um ... what can I say "weird" and I felt it was ok to ask questions about that and then he became very denying as he came to me for problems with his knee. And one week later he was hospitalized with suicide attempt where he had cut 
himself in the arm: I hadn't seen that coming even though I thought that something was wrong with that man, so it was not possible to pressure him to talk about it, but I sort of invited him to an appointment or open dialog if he needed it, so ... and when he came to me for a consultation after the suicide attempt and told that he did it because of the knee problem. I really didn't believe that.

\subsubsection{Masking Actual Problems}

Sometimes, patients contacted GPs with somatic symptoms that later turned out to be psychological, as illustrated in this quote:

".. very often there are patients that come to us with a defined problem that is not the case, and after a while we learn that they can come to us with a slow concrete banal ailment. It can be something physical, like:

"I have pain somewhere and wonder what it might be." in the way that one senses that it may not only be this, but think that they have scheduled an appointment for something they come up with to make contact...

"It might be that they make up something to avoid the receptionist"... And that they don't want to tell others than the GP.. maybe even difficult to talk about with the GP and I think as a GP you should be very aware of that... If you can sense it and ask that question.

\subsection{Cues and Concerns: Patient Disclosure of Problems}

The question about how the GPs revealed mental health problems and suicide ideation depended partly on unexplainable factors and in what manner the patients communicated them. The patients' cues and concerns had to be recognized and identified.

Three categories of communicating suicidality emerged from the data: verbally explicit, indirectly verbal and non-verbally. In some rare cases, the GPs had tried but found it impossible to enter the patient's inner thoughts, and one described this as a "black box".

\subsubsection{Explicit Verbal Implication Example}

.. I have called the acute team several times when I have suspected that something has to be done immediately, for example I had a woman in her fifties, very up-and coming resourceful woman who said: "If nothing happens now I will drive into a wall. or I am like complete out now..um.. It has been psychiatric problems all the way, and she was like all out now.. I called, then I called the team right way, and they took care of her and initialized hospitalization.

And if someone is so open and direct it is much easier to deal with, it's difficult when someone just turn their back and pretend that everything is ok, and it isn't. That is difficult.

"Because if you don't ask and dig into it ... um.. then you often will only get information about something that might be rooted in something deeper, and so it is not always you get it in a twenty-minute consultation, but then, if I sense it, in this case it is important to seize, I often end the conversation by having a dialog with the patient in order to have a new conversation ... and they think of that as positive and I schedule one hour to talk more about the psychiatric part, then it is incredible how much that is disclosed and revealed because then we are both prepared to talk and if you dig deeper and ask you will find out a lot".

\subsubsection{Indirect Verbal Implication}

The "door handle" was when the consultation was over, the patient was about to leave and, on the way out, held the door handle and said "by the way ... ." or accidently dropped a quote at the end of a consultation like "By the way I need a referral to a psychologist". 


\subsubsection{Non-Verbal Implication}

There was an agreement that the level of training and experience increased the ability to sense and respond to cues given by the patients and see beyond "that ear" or "that knee that hurt". This ability also increased in line with the duration of the doctor-patient relationship. The GPs with more experience followed some of the patients for several years and followed life events like marriage, pregnancy or divorce.

When they knew the patient, it was easier to recognize whether their illness behavior had altered, and they immediately understood that something serious was going on.

In addition, when they followed a patient regularly, it was possible to recognize a shift in the patient's behavior that indicated a need for more thorough assessment.

The GPs also described how the recognition of patterns allowed them to diagnose different diseases. Sometimes it was undoubted that a depressed patient arrived at the office.

However, in some of the cases, the GPs could not quite explain why or how they suspected or sensed that there was something more serious than what the patients communicated verbally. They referred to this feeling as follows:

"If I am sitting with a bad gut feeling", "Sometimes I just get concerned" and "You have to be aware of whether this trigger any tears or feelings in yourself".

One of the GPs described that if a patient was "blurry", it was possible to choose the degree of involvement, as they were not blamed and, to some extent, could choose how much they should engage and follow cues. The GPs could also have a "bad day", often in combination with a lack of time.

One of the GPs described that she was sometimes worried:

"But there are these youngsters, like they are really ill and stuff ... they don't get in touch to get help and you have to grab a hold of yourself and I get very worried for them if they don't pick up the phone when I try to call them several times".

Furthermore, the GPs described that they had to be able to "keep out their antennas" and be curious. Some emphasized the importance of being primed, and one of the GPs explained how a colleague with high expertise in depression in adolescents "could spot a depressed youngster behind almost every corner", illustrating the fact that knowledge and expertise increased their ability to recognize psychological problems.

The GPs were extra aware when risk factors for suicide were present in addition to psychosocial problems, such as being a young male or previous suicide attempts.

Some described that they sometimes "got a hunch" about the patient's somatization and tried to find out whether these somatic symptoms were due to psychological problems:

"It is most often the boys I have noticed, like around 30 to 45 years. In these age groups is it a lot ... I don't know if I is because I am female doctor that they are maybe don't able to talk about, they somatize a lot and some come and say that they have migraine and I say that if you have so much migraine maybe we have to figure it out, and then it actually was anxiety..."

\subsubsection{Non-Explicit Patients and the "Black Box"}

In cases where a patient had attempted or died by suicide, the GPs said that they used a considerable amount of time to figure out whether there was a sign of anything during the last contact with the patients preceding the suicide. However, even if they knew the patient well, it was not possible in retrospect to find any cues or warning signs that either could explain or predict that this patient was going to end his or her life:

"I follow up if I have a feeling that there is something and then I call, but it was to late, but I don't know really, it isn't always possible to do something ... "

"I think that among some patients it is impossible to enter the patients inner "black box", and in some cases it is not possible to crack it, they never reveal their inner thoughts." 


\subsection{Clarifying Phase: Tuning into the Essence of the Problem and Forming an Alliance}

"I think that ... as a new general practitioner ... you have to do a thorough anamneses and get to know the patient and that is the positive thing about being a general practitioner, that you know the patient and can schedule a new appointment soon and you can tell them that you can call the next day ... it is a low threshold to come back, the MADRS is useful, because then you get to catch sleep, anxiety and different things in a good way.."

To form an adjustive alliance toward agreement about a depressive state of mind and possible suicide, ideation emerged as an important factor in their work. However, it was not always easy to find an opening where they could ask about suicide ideation.

The GPs gave examples of patients that were resistant to accept that they were depressed or reveal their extent of distress. They often had to circle to a common understanding of if the patients were blurry or vaguely indicated more serious psychological problems. To dig deeper into the patient's situation and problems, they asked about practical daily life activities with questions like "How does your laundry room look like?" or "Do you get enough sleep at night?"

This was a careful process where the GPs had to establish agreement and consensus with the patients about whether symptoms like fatigue or sleep disturbance could indicate underlying depression rather than, for example, somatic conditions:

“... But the ones that don't quite understand that they might have a depression, you have to be careful and take it slowly to get them to follow and agree to the fact that this might be due to depression."

"You cannot just throw a depression diagnose into their lap"

Several of the GPs described cases where female patients in particular seemed to prefer a somatic diagnosis instead of a psychiatric one. Examples included myalgia encephalomyelitis or hypothyroidism:

"Of course, we examine all kinds of possible explanations, with blood samples, cardiologist, and neurologist, but if we don't find anything, we have to cut the crap and find the real problem"

For looping patients, because it was not possible to establish a further treatment plan without a common understanding of the problem, the GPs sometimes experienced some of the patients as being time-consuming and frustrating, especially when patients refused to listen to their advice and it was impossible to develop any further progress:

"So why can you not listen to me for the twentieth time, instead of your friend that happened to read about this in a magazine or at Google ..."

Two of the major qualities that promoted and inhibited the forming of an alliance and the process of identification were sufficient time and how it was communicated. Factors that hindered constructive communication with the patients were to avoid eye contact, write on the computer, look at a watch or show restlessness, such as by shaking the leg while the patient talked. The importance of waiting for the patient to talk and endure latency was underlined together with an understanding and interested behavior:

"We have to look them in the eye, endure latency and show them interest and understanding"

In cases where it was impossible to obtain an alliance with the patients, the GPs acknowledged this and referred to other health care services.

\subsection{Identification of Suicide Ideation}

The use of the Montgomery and Aasberg Depression Rating Scale (MADRS) was commonly mentioned to assess depression and suicidality. The GPs were satisfied with this scale that was in the electronic medical system, and they often incorporated the questions into a conversation. This enabled them to obtain a broader understanding and map out the severity of the depression. One of the GPs pointed out that the question about suicidality 
was the last one, which underpinned that they needed to establish an alliance and an agreement before asking that question.

"We don't ask about suicidal thoughts when they get a prescription on p-pills you know"

"We bring it up after a while, it is a reason why that question is the last one I MADRS"

\subsection{Preventive Interventions in General Practice}

The patients arrived their GPs office with different problems and needs. One quote was particularly interesting and described the essence of the study's aim:

“..But in the aftermath some patients have come to me and said: That thing you said at that point, or what you did then, that saved my life"

What was this that was said or done? The GPs explained how they initiated primary preventive measures that might interrupt and stop the development of a suicidal crisis. Examples included sleeping problems, life crisis, medication for pain, sick leave and referral to a specialist. They frequently scheduled and often prolonged consultations:

Some try without a specialist and then we try other things, routines, sleep, eat properly ... . just come to talk and figure out things. It is often helpful to come and talk about and turn around on things, it is often job stuff. Many are unsatisfied at work, but then you must do something about it ... I am a mother, a coach a psychologist ...

One important phenomenon that emerged was the combination of providing the patients with hope, safety and commitment, with phrases such as the following:

"I will help you getting through this", "I will be here for you", "I can help you get through this", "I am good at this", "It is not dangerous", and "It will be better when we have found out of these problems that you have" "I will take care of you, I am good at this".

The GPs underlined that they were available, often provided the patients with a telephone number and encouraged contacting them if a crisis emerged and, in some cases, also encouraged doing so outside of office hours. If the patient did not arrive for the scheduled appointment, they worried and telephoned them or contacted their significant others:

\section{"If I have a feeling that something is wrong, I call them"}

Even out of the scope for this study, some necessary interventions outside general practice should be mentioned. The GPs described measures where they referred to a specialist or higher levels of health care. One GP said that she knew the specialties of some of the psychologists (e.g., in personality disorders or couples therapy) and then recommended a more customized treatment. The GPs also underlined that if they followed the patient over time or there was a more acute situation, they referred to specialist health care, like acute ambulant teams or psychiatric outpatient clinics. If there was an acute crisis, they had to let all other patients wait and arrange for hospitalization.

\section{Discussion}

\subsection{Summary of Main Findings}

The main findings described the pathways into how GPs disclosed suicidality among their patients. Furthermore, the steps in the process, from how the initial diagnoses and symptoms were investigated together with the cues and concerns to how they formed alliances and tune into common agreements with their patients, were revealed. The data also showed insight into how the GPs provided patients with hope and safety by being available and committed. Customized interventions that might interrupt the process and might lead to subsequent suicidal behavior were described. The examples were small steps, like a shorter period of sick leave, keeping regular contact during a life crisis or treating and referring to help with chronic pain that was closely associated with suicidality [10]. In 
line with previous research, it was essential to establish a working relationship in which the patient felt listened to and understood [11].

\subsection{Strengths and Limitations of the Study}

This study is presented in line with the COREQ statement [9], and the current qualitative research findings extend and build a picture of the phenomenon and add knowledge to this particular area of general medicine, where researchers often have focused on retrospect and used quantitative registry data.

The first limitation was the sample size; however, saturation was achieved in the main findings. The second limitation was the recruitment (convenience plus snowball sampling), as the respondents could be less representative of GPs in general (i.e., more informed or interested about suicide-related topics compared with average people).

\subsection{Comparison with the Existing Literature}

It is difficult to determine the prevalence of suicidal ideation in general practice. One study from the USA found that suicide was discussed with $11 \%$ of the patients that had reported having suicidal thoughts before the consultation without the GP knowing [12]. In a Norwegian study, $25 \%$ of the patients reported having suicidal thoughts, and approximately $50 \%$ talked with their GPs about this during their last contact before they attempted suicide [13]. A psychological autopsy study of 571 suicides demonstrated that $22 \%$ of the patients that had been in contact with health care services less than four weeks before the suicide had discussed suicide ideation, whereas $21 \%$ of those who ended their lives the same day had talked about it [14]. The findings in our study showed that the GPs sometimes were surprised and were not able to understand why their patient ended his or her life. This is in line with the findings that the GPs often found a patient's suicide shocking and surprising [15]. It is important to bear in mind that patients might conceal their thoughts or plans or, on the contrary, talk about suicidal thoughts without having serious intention to end their lives [16]. In a study of 61 completed suicides among patients in general practice, Milton et al. demonstrated a low rate of risk assessment and limited knowledge of the patient's lifestyle. They also found that only 1 out of the 61 GPs felt that the suicide could have been prevented [2]. However, there is a distinction between whether they perceived them as impossible to predict or impossible to prevent. In our study, some of the GPs that had experienced suicide among patients said that they had really tried to think of any signs or cues that they should have discovered during the last consultation. However, they could only speculate as to why this had happened.

In addition, as pointed out by Sakinofsky, "A truly suicidal patient has no interest in betraying his intentions to someone likely to impede them" [17].

The findings described how the GPs provided their patients with hope and ensured that they could help them. Hopelessness has been pointed out as a strong and stable factor associated with suicidality $[18,19]$. Interventions that decrease pessimism and provide hope might therefore be useful in all levels of suicide prevention initiatives.

\subsection{Clinical Implications}

The patients in general practice with unrecognized symptoms were an interesting finding. For health care personnel, it might be difficult to understand or they might forget that symptoms that are well known associated to be associated with, for example, depression are not recognizable to all people. The fact that many members of the public cannot recognize specific disorders or different types of psychological distress is therefore important to bear in mind. The evidence from surveys in several countries about the decencies in mental health literacy among the public, such as how to recognize the development of a mental disorder in contrast to physical illness, was pointed out by Jorm [20]. The use of screening instruments can disclose suicidal thoughts, and the Montgomery and Aasberg Depression Rating Scale (MADRS) was commonly mentioned by the GPs. Even though screening instruments cannot replace a clinical interview, it might be a useful 
supplement. Many of the GPs incorporated it into the conversation or used it to tune into the question about suicidality. Such instruments can also be useful to measure and follow the development of depressive and suicidal symptoms over time. In a study where suicide risk was incorporated in a standardized electronic tool for psychosocial history among adolescents, the frequency of suicide risk assessments doubled [21]. The referral rates increased correspondingly, but it was not investigated to what extent the young people were followed up on and received treatment or whether this contributed to any health benefits. Such findings can therefore not provide evidence about a direct effect on suicidal behavior. However, in some cases, it might lead to improved health services for patients and thus an indirect preventive effect.

In our study, the GPs that had experienced suicide among their patients said that they had really tried to think of any signs or cues that they should have discovered during the last consultation. However, they could only speculate why this had happened. A Danish study of GPs showed that the experience of a patient's suicide had a substantial emotional effect on them. Examples included feelings of guilt and failure. Furthermore, if patients had contacted them about physical symptoms and they did not diagnose any suicidality, this led to considerable self-scrutiny [15]. In our study, some explained how they sometimes revealed mental problems among patients that were somatizing, such as with headaches. They also treated and dug deeper into physical illness that was associated with worries and psychological stress. Adult studies on the non-disclosure of psychological problems suggest that patients contribute to non-detection by presenting their distress as somatic rather than emotional [22]. Further, recognition of psychiatric distress in primary care is reduced with increasing levels of somatization [23].

It is important to underline that even if the self-scrutiny in some cases may lead to increased knowledge, the retrospective perspective might contribute to curbing the personal burden for health care personnel and the fear of being blamed. To bear in mind the fact as mentioned earlier that not all will tell anyone likely to intervene in their suicide plans [17]. One of the main findings is the amount of time needed to reveal, address and prevent suicidality in all the steps of the process. This is an important factor to recognize for clinicians and policy makers, especially since these experiences have been mirrored in previous research from patients' views, which demonstrated that worrying about wasting a GP's time is a complex barrier to help-seeking. GP time and resource scarcity, symptom gravity, appointment etiquette and previous GP interactions contributing to increasing these worries. Friendly GP relationships, economic reasoning and a focus on the GP's responsibilities as a medical professional reduce this worry [24].

\subsection{Implications for Future Research}

This study has shown insight into a GP's work and clinical practice. There is no evidence to measure the extent to whether their role and work contribute to the prevention of suicidal behavior. However, the findings should be examined in more detail based on the patient's views. Patients can mirror these findings and elaborate on what GPs said or did, or on the contrary, what they did not say or could have done differently. It is probably also useful for the field and for theoretical training to investigate these current findings further in order to have a better understanding of what GPs are comfortable and confident with, where they struggle and where they experience meaningful outcomes.

The empirical literature with this primary focus is scarce. Researchers should consider the experiences of patients in life crises or depressive states who have not attempted suicide and provide insight to understand and inform clinical interventions. Furthermore, additional insight into why some people are willing to discuss suicidal thoughts and plans while others are a black box is useful knowledge. People that have survived suicide attempts would probably provide valuable information about the events and communication preceding the attempt. Another finding that should be pursued and further investigated is the prevalence of mental health literacy in relation to suicidality in the public and in health care services. It is important to raise awareness in the public about the GP's role in help 
seeking when a crisis emerges. Furthermore, whether it is stigma, other phenomena or a combination that contributes to the resistance against being diagnosed with psychiatric illness should be focused on in public health research initiatives.

\section{Conclusions}

In this study, we explored the experiences of GPs and their views of suicide prevention in their practices. Many patients arrive with diffuse symptoms, and if they sense or understand that there might be a risk of suicidality, they must firmly develop a common understanding with the patients. Small steps to help, like more frequent consultations and a personal commitment together with creating hope and safety, might in some cases help and avoid any further escalation of a crisis.

There are several factors that are important, but time seems to be crucial to both identify and follow up with sufficient measures.

Author Contributions: Conceptualization, methodology, interviewing, project administration and funding acquisition, T.K.G.; validation, transcripts, formal analysis and writing, M.S. and T.K.G. Both authors have read and agreed to the published version of the manuscript.

Funding: This research was funded by The Dam Foundation, the Council of Mental Health in Norway (grant number 2015/ F05106) and the Research Council of Norway (grant number 288731).

Institutional Review Board Statement: The study was conducted according to the guidelines of the Declaration of Helsinki and the Personal Protection Agency at Oslo University Hospital. Approval from an ethics committee was not necessary.

Informed Consent Statement: Informed consent was obtained from all subjects involved in the study.

Data Availability Statement: The full dataset is transcribed in the Norwegian language but can be made available upon reasonable request.

Acknowledgments: The authors would like to thank the GPs that participated in the interviews and shared their experiences and perceptions, and further thanks to the Department of General Practice at the University of Oslo for suggestions and help with recruitment.

Conflicts of Interest: The authors declare no conflict of interest. The funders had no role in the design of the study; in the collection, analyses, or interpretation of data; in the writing of the manuscript, or in the decision to publish the results.

\section{References}

1. Mann, J.J.; Apter, A.; Bertolote, J.; Beautrais, A.; Currier, D.; Haas, A.; Hegerl, U.; Lonnqvist, J.; Malone, K.; Marusic, A.; et al. Suicide prevention strategies: A systematic review. JAMA 2005, 294, 2064-2074. [CrossRef]

2. Milton, J.; Ferguson, B.; Mills, T. Risk Assessment and Suicide Prevention in Primary Care. Crisis 1999, 20, 171-177. [CrossRef]

3. Sakinofsky, I. The Family Doctor and the Prevention of Suicide. Can. Fam. Physician Med. Fam. Can. 1982, 28, 1606-1610.

4. Statistics Norway. Available online: https://www.ssb.no/en/statbank/table/10141/tableViewLayout1/ (accessed on 5 August 2021).

5. Luoma, J.; Martin, C.E.; Pearson, J.L. Contact with Mental Health and Primary Care Providers Before Suicide: A Review of the Evidence. Am. J. Psychiatry 2002, 159, 909-916. [CrossRef]

6. Michel, K.; Runeson, B.; Valach, L.; Wasserman, D. Contacts of suicide attempters with GPs prior to the event: A comparison between Stockholm and Bern. Acta Psychiatr. Scand. 1997, 95, 94-99. [CrossRef]

7. Murphy, E.; Dingwall, R.; Greatbatch, D.; Parker, S.; Watson, P. Qualitative research methods in health technology assessment: A review of the literature. Health Technol. Assess. 1998, 2, 1-274. [CrossRef]

8. Pope, C.; Ziebland, S.; Mays, N. Qualitative research in health care: Analysing qualitative data. BMJ 2000, 320, 114-116. [CrossRef] [PubMed]

9. Tong, A.; Sainsbury, P.; Craig, J. Consolidated criteria for reporting qualitative research (COREQ): A 32-item checklist for interviews and focus groups. Int. J. Qual. Health Care 2007, 19, 349-357. [CrossRef]

10. Tang, N.K.Y.; Crane, C. Suicidality in chronic pain: A review of the prevalence, risk factors and psychological links. Psychol. Med. 2006, 36, 575-586. [CrossRef] [PubMed]

11. Buszewicz, M.; Pistrang, N.; Barker, C.; Cape, J.; Martin, J. Patients' experiences of GP consultations for psychological problems: A qualitative study. Br. J. Gen. Pract. 2006, 56, 496-503.

12. Vannoy, S.D.; Robins, L.S. Suicide-related discussions with depressed primary care patients in the USA: Gender and quality gaps. A mixed methods analysis. BMJ Open 2011, 1, e000198. [CrossRef] 
13. Grimholt, T.; Jacobsen, D.; Haavet, O.; Sandvik, L.; Jorgensen, T.; Norheim, A.; Ekeberg, O. Structured follow-up by general practitioners after deliberate self-poisoning: A randomised controlled trial. BMC Psychiatry 2015, 15, 245. [CrossRef]

14. Isometsä, E.; Heikkinen, E.M.; Marttunen, M.J.; Henriksson, M.M.; Aro, H.M.; Lönnqvist, J.K. The last appointment before suicide: Is suicide intent communicated? Am. J. Psychiatry 1995, 152, 919-922. [CrossRef] [PubMed]

15. Davidsen, A.S. And then one day he'd shot himself. Then I was really shocked: General practitioners' reaction to patient suicide. Patient Educ. Couns. 2011, 85, 113-118. [CrossRef] [PubMed]

16. Freedenthal, S. Assessing the Wish to Die: A 30-Year Review of the Suicide Intent Scale. Arch. Suicide Res. 2008, 12, 277-298. [CrossRef]

17. Sakinofsky, I. Preventing suicide among inpatients. Can. J. Psychiatry 2014, 59, 131-140. [CrossRef] [PubMed]

18. Kuo, W.H.; Gallo, J.J.; Eaton, W.W. Hopelessness, depression, substance disorder, and suicidality-a 13-year community-based study. Soc. Psychiatry Psychiatr. Epidemiol. 2004, 39, 497-501. [CrossRef] [PubMed]

19. Beck, A.T.; Steer, R.A.; Beck, J.S.; Newman, C.F. Hopelessness, depression, suicidal ideation, and clinical diagnosis of de-pression. Suicide Life Threat. Behav. 1993, 23, 139-145. [CrossRef]

20. Jorm, A.F. Mental health literacy: Empowering the community to take action for better mental health. Am. Psychol. 2012, 67, 231-243. [CrossRef] [PubMed]

21. Wintersteen, M.B. Standardized Screening for Suicidal Adolescents in Primary Care. Pediatrics 2010, 125, 938-944. [CrossRef]

22. Olde Hartman, T.; van Rijswijk, E.; van Ravesteijn, H.; Hassink-Franke, L.; Bor, H.; van Weel-Baumgarten, E.; Lucassen, P. Mental health problems and the presentation of minor illnesses: Data from a 30-year follow-up in general practice. Eur. J. Gen. Pract. 2008, 14, 38-43. [CrossRef] [PubMed]

23. Kirmayer, L.J.; Robbins, J.M.; Dworkind, M.; Yaffe, M.J. Somatization and the recognition of depression and anxiety in primary care. Am. J. Psychiatry 1993, 150, 734-741. [PubMed]

24. Cromme, S.K.; Whitaker, K.L.; Winstanley, K.; Renzi, C.; Smith, C.F.; Wardle, J. Worrying about wasting GP time as a barrier to help-seeking: A community-based, qualitative study. Br. J. Gen. Pract. 2016, 66, e474-e482. [CrossRef] [PubMed] 